\title{
La formation des professionnels de la santé à l'heure de la pandémie de COVID-19. Réflexions, enquêtes, partage de pratiques et retours d'expériences
}

\author{
Healthcare professionals education at the time of the COVID-19 pandemic. \\ Reflections, surveys, sharing of practices and experience feedbacks
}

Ce dernier numéro du millésime 2020 de Pédagogie Médicale est entièrement consacré au dossier thématique qui réunit une première série de contributions reçues en réponse à l'appel éditorial exceptionnel, que la rédaction avait lancé au printemps dernier dans le contexte de la pandémie de COVID-19.

Alors qu'elle est toujours active près d'un an après son émergence, cette pandémie constitue un évènement planétaire et séculaire qui bouleverse en profondeur, sans doute durablement, l'organisation de nos sociétés.

Concernant la formation des professionnels de la santé, la crise sanitaire induite par cette pandémie a d'abord mis à l'épreuve nos systèmes et personnels de santé, qui ont dû inventer extemporanément des solutions adaptatives inédites. Sur un autre plan, le confinement imposé aux personnes a conduit, dans un premier temps, à fermer les universités et les instituts de formation, puis à les réouvrir, moyennant cependant de multiples contraintes organisationnelles, liées à la nécessité perçue de maintenir durablement des barrières de distanciation physique.

Parce que la santé est son objet et parce qu'elle s'effectue non seulement dans les institutions mais aussi, pour une large part, en milieu de soins, qu'il soit hospitalier ou ambulatoire, l'éducation médicale - entendue au sens générique comme concernant la formation de tous les professionnels de la santé - a dû ainsi relever de multiples défis nouveaux, en recourant à des interventions vicariantes. À ce jour, les conséquences en sont pour la plupart spéculatives. On doit cependant faire l'hypothèse que certaines d'entre elles pourraient durablement modifier les dispositifs curriculaires et pédagogiques concernés.

Tel est notamment le cas des dispositifs de formation à distance, qui se sont déployés et substitués aux dispositifs organisés en présence, pour assurer ce qu'il est désormais convenu de nommer «continuité pédagogique». Pour ce faire, ils ont eu massivement recours à la mise en ligne de ressources didactiques et aux divers échanges à distance, synchrones ou asynchrones, permis par les plates formes numériques. Illustrant les nouveaux environnements d'enseignement et d'apprentissage, dont une contribution de Feigerlova et ses collègues présente un panorama synthétique [1], plusieurs communications brèves publiées dans ce numéro y sont consacrées[2-8]. Elles montrent, par exemple, comment des formats d'intervention pédagogique foncièrement pensés pour des interactions en présence, tels que les séances d'apprentissage du raisonnement clinique (ARC) [6] ou les examens cliniques objectifs structurés (ECOS) [5], peuvent être transposés dans des dispositifs à distance, au prix de certaines adaptations. Elles montrent à la fois toutes les potentialités bien réelles de ces nouveaux outils mais aussi certaines de leurs limites, dont il faudra, le moment venu, faire loyalement l'inventaire et l'analyse, en examinant de façon critique les promesses qui auront été tenues et les nouveaux problèmes qui seront apparus.

Un éditorial de Thierry Pelaccia et ses collègues problématise ces différents enjeux et questionne certaines des prémisses de l'enseignement à distance, en invitant notamment à ne pas confondre les buts et les moyens [9]. D'aucuns pourraient en effet être définitivement séduits par l'apparente et immédiate efficience des outils aujourd'hui disponibles, et tentés d'y voir une préfiguration prometteuse de l'évolution inéluctable à laquelle les curriculums seraient désormais astreints, au regard d'un avenir numérique fantasmé, nécessairement radieux. D'autres en redoutent les effets, notamment en lien avec le risque d'une dénaturation foncière de la relation pédagogique qu'ils pourraient engendrer, source, entre autres, de nouvelles discriminations et inégalités, par exemple dans le cadre des procédures d'admission aux études de santé, comme l'analyse l'une des contributions [10].

Les principes et les outils de l'enseignement à distance se sont progressivement développés pour rapprocher des enseignants, des formateurs et des étudiants qui étaient éloignés en raison de diverses contraintes, notamment géographiques. Il serait en l'occurrence paradoxal qu'ils soient désormais utilisés pour mettre à distance des étudiants bien présents, au seul prétexte de divers avantages matériels escomptés (économies financières, 
gain de temps, etc.), en confondant tout particulièrement des activités qui relèvent de niveaux différents, que sont respectivement celui des interfaces (niveau technologique), celui des interactivités (niveau cognitif) et celui des interactions (niveau de l'engagement des personnes). De telles méprises constitueraient à vrai dire un authentique fétichisme, au sens où ce terme désigne le processus par lequel des artefacts, c'est-à-dire des objets fabriqués - en l'espèce les ressources technologiques éducatives -, deviennent surestimés, en finissant par être utilisés pour eux-mêmes, ayant perdu le lien avec les échanges sociaux au service desquels ils avaient été élaborés - en la circonstance, ceux qui sont constitutifs de la relation pédagogique-, en réifiant du même coup ces derniers. À tous ces égards, il semble opportun de rappeler toute l'actualité que conserve l'avertissement que formulaient Grabe et Grabe dès 1996: «Just remember: in most cases, effective teaching with technology is effective teaching by any means»; «Souvenez-vous de ceci: dans la plupart des cas, un enseignement efficace avec le concours des technologies est d'abord un enseignement efficace quels que soient les moyens utilisés » (traduction libre) [11].

Les conditions de la formation en contexte de stage ont par ailleurs été fortement transformées. Ici ou là, des étudiants ont été projetés dans des fonctions habituellement dévolues à des médecins séniors ou ont été enrôlés comme auxiliaires des professionnels de santé, parfois dans des tâches valorisantes, qui ont élargi leurs expériences d'apprentissage, mais parfois dans des tâches subalternes. Ailleurs, ils ont été tout simplement oubliés ou évincés des milieux de stages, tantôt au motif allégué qu'il ne fallait pas les exposer au risque infectieux mais quelquefois, sans qu'évidemment cela soit exprimé ainsi, parce qu'ils étaient soudain devenus inutiles, voire «encombrants», dans des organisations de soins soudainement déstabilisées. Plusieurs retours d'expérience rapportent les adaptations ou initiatives qui ont été mises en œuvre, par exemple pour faire face au risque infectieux très spécifique de la formation pratique en dentisterie [12], au bloc opératoire [13] ou au service des urgences [14], ou encore pour explorer des thèmes nouveaux, comme celui du remplacement en médecine générale [15], ou pour approfondir des situationsproblèmes plus complexes en pédiatrie [16].

En lien avec ces problématiques, plusieurs contributions insistent judicieusement sur la nécessaire primauté que devraient avoir les finalités des dispositifs de formation par rapport à leurs formats. Elles invitent à considérer, à cet égard, que le contexte de la crise sanitaire est à la fois, certes, une source de difficultés importantes, comportant le risque de mise en ouvre de modalités «dégradées», mais aussi une opportunité historique privilégiée à saisir pour dépasser certains conservatismes et mettre en place des innovations porteuses de sens.

Alors que de nombreuses sessions d'examen ont été ajournées et différées, et que d'autres activités d'évaluation ont parfois été mises en œuvre sous des formats improvisés, à la pertinence et à la fiabilité incertaines,
Valérie Campillo et ses collègues [17] invitent ainsi à se réapproprier les finalités et les principes fondamentaux de l'évaluation des apprentissages, pour mieux en repenser les modalités adaptatives dans le cadre de dispositifs à distance. Pour leur part, dans une tribune, Ahmed Maherzi et ses collègues [18] réaffirment la pertinence du paradigme de la responsabilité sociale des institutions de formation en santé pour réorienter les finalités des curriculums autour de problématiques sociales, dont le caractère crucial a été révélé par les différentes tensions observées dans presque tous les pays, relativement à la gestion mal anticipée des problèmes sanitaires. Dans cette veine, une communication brève illustre comment la situation a été exploitée pour introduire des objectifs de santé publique et des thèmes sociétaux en odontologie [19], une autre comment des initiatives de recherche participative impliquant les étudiants ont pu être initiées autour de la question de leur responsabilité sociale [20], et une autre encore en quoi le dispositif de formation par concordance s'avère particulièrement fécond pour conduire des expériences d'analyse de pratique lorsqu'il s'agit d'affronter les multiples et mouvantes incertitudes d'une crise sanitaire [21].

Nonobstant leur richesse et leur diversité, ces différentes contributions n'épuisent évidemment pas les réflexions à conduire autour des problématiques éducationnelles liées à la formation des professionnels de la santé, induites, révélées ou exacerbées par le contexte de la pandémie de COVID-19. Il convient d'une part de rappeler que, tel qu'il était formulé, l'appel à contribution entendait favoriser l'actualité des projets d'articles, au regard de la réactivité et de l'inventivité dont les institutions et leurs acteurs avaient dû faire preuve lorsqu'il s'est agi de mobiliser diverses ressources, dans le contexte contraint de l'irruption de la crise sanitaire, et de les exploiter pour adapter, voire développer de novo, un certain nombre de dispositifs curriculaires et de dispositifs pédagogiques d'enseignement, d'apprentissage et/ou d'évaluation. Dès lors, il était explicitement assumé que les travaux reçus puissent privilégier une approche pragmatique, et ne pas forcément satisfaire toutes les exigences qui auraient été requises si les intentions avaient été d'une autre nature, par exemple de procéder à une revue méthodique et systématique de la littérature. Passé le moment de l'urgence qu'il y avait à opérationnaliser la continuité pédagogique, il convient maintenant de poursuivre l'examen critique approfondi des multiples questions didactiques ou pédagogiques qui sont posées, à la fois au plan méthodologique mais aussi au plan épistémologique.

Ainsi, les valeurs en jeu dans les curriculums implicites, si puissantes pour soutenir les processus de socialisation et de construction identitaire professionnelles, mériteraientelles sans doute d'être interrogées, comme par exemple celles liées à la figure du héros, subitement projetée sur les soignants et qui, assurément, exalte certaines vocations et sublime certaines motivations, en même temps qu'elle amplifie les risques psycho-sociaux, en sur-sollicitant les exigences de performance chez les étudiants et en leur interdisant la reconnaissance de leur propre vulnérabilité. 
Ainsi en va-t-il aussi des questions éducationnelles liées à la culture scientifique des professionnels de la santé. Des polémiques parfois confuses se sont développées autour de l'évaluation scientifique des stratégies thérapeutiques à développer face à un problème de santé subitement émergeant, et autour de la légitimité à les mettre en œuvre dans le cadre d'une pratique soignante, alors que persistaient des incertitudes - le cas de l'hydroxychloroquine étant à ce point de vue emblématique. Elles ont montré combien de nombreux médecins, y compris ceux ayant une pratique régulière de la recherche clinique, étaient à cet égard dépourvus de repères en épistémologie ou en philosophie des sciences, n'ayant pour horizon indépassable, en lien avec les scotomes de leur formation initiale à cet égard, que celui offert par la médecine expérimentale ou par l'épidémiologie clinique, dans une conception parfois très simplificatrice des notions de preuve et de démonstration, auxquelles on réduit souvent la perspective de l'evidence-based medicine (EBM). Le moment est sans doute opportun pour intégrer authentiquement dans la formation des professionnels de la santé, quand ils n'y sont pas déjà, les éclairages de nombreux champs scientifiques et notamment ceux issus des sciences humaines et sociales, comme l'anthropologie de la santé, la sociologie ou l'économie politique en santé, ou encore la philosophie pratique et morale et la philosophie des sciences appliqués à la santé, rejoignant ainsi des perspectives contemporaines comme celle de la promotion de la santé, telle qu'elle est énoncée depuis 30 ans dans la charte d'Ottawa [22].

Dans ce contexte, poursuivant la contribution qu'elles souhaitent apporter à l'intelligibilité de la situation très inédite à laquelle sont confrontées les institutions de formation des professionnels de la santé, ainsi que leurs différents acteurs, au sein de la communauté internationale francophone, la Société internationale francophone d'éducation médicale (SIFEM) et son organe officiel d'expression, la revue Pédagogie Médicale, ont décidé de prolonger l'appel exceptionnel à contributions COVID-19 jusqu'au 30 juin 2021. Les projets d'articles qui seront retenus feront l'objet de rubriques éditoriales thématiques complémentaires, voire d'un deuxième numéro spécial, dans le courant de l'année 2021.

Jean JOUQUAN Rédacteur en chef

*Mailto : jean. jouquan@univ-brest.fr

\section{Références}

1. Feigerlová E, Hani H, Lopes R, Zuily S, Braun M. COVID19: Quelques pistes pour un nouvel environnement d'enseignement et d'apprentissage en contexte de mise à distance des enseignants et des étudiants en médecine. Pédagogie Médicale 2020;21:187-193.
2. Scherly D, Nendaz M, Bouvet G, Audétat M-C, Baroffio A. Transition vers une formation à distance pour les professionnels de santé en temps de COVID-19: du scénario pédagogique à une analyse SWOT. Pédagogie Médicale 2020;21:207-210.

3. Picchiottino P, Paignon A, Bachmann B, Elmaleh M. Simulation interprofessionnelle en visio-conférence. Pédagogie Médicale 2020;21:211-213.

4. Descôteaux A, Jackson M, Vanier M-C. Quand les patients formateurs prennent le relais: transfert d'ateliers sur la collaboration interprofessionnelle en ligne en temps de COVID-19. Pédagogie Médicale 2020;21:215-217.

5. Meister V, Junod-Perron N, Geoffroy F, Paignon A. Examen clinique objectif structuré (ECOS) formatif avec patient simulé et rétro-action par un pair en visioconférence. Pédagogie Médicale 2020;21:219-221.

6. Massart V, Buret L, Giet D, Belche J-L, Dory V. Séance d'apprentissage du raisonnement clinique (ARC) à distance. Pédagogie Médicale 2020;21:223-226.

7. Charfi R, Cheikhrouhou S, Labbene I, Jouini M. Enseignement médical en ligne solidaire durant la pandémie de COVID-19. Pédagogie Médicale 2020;21:241-243.

8. Ben Amor A, Charfi R, Ben Hammouda S, Jouini M. Enseignement de la gynécologie-obstétrique aux étudiants en médecine en temps de pandémie. Pédagogie Médicale 2020;21:245-247.

9. Pelaccia T, Demeester A, Charlin B, Denef J-F, Gagnayre $\mathrm{R}$, Maisonneuve $\mathrm{H}$ et al. Le déploiement de la formation à distance au sein des facultés de médecine dans le contexte de la crise sanitaire liée à la COVID-19 : et après? Pédagogie Médicale 2020;21:173-174.

10. Leduc J-M, Bourdy C, Loye N. Les répercussions liées à la COVID-19 sur les processus d'admission en médecine. Pédagogie Médicale 2020;21:199-202.

11. Grabe M, Grabe C. Integrating technology for meaningful learning. Boston (MA): Houghton Mifflin Company, 1996.

12. Nguyen Ngoc C, Kontaxis KL, Epure E. L'enseignement de la médecine dentaire en période de pandémie COVID-19. Pédagogie Médicale 2020;21:249-251.

13. Baudin F, Yung F, Toure T, Lesage S. Formation des professionnels au bloc opératoire pédiatrique en contexte COVID-19: comment répondre aux inquiétudes des différents acteurs? Pédagogie Médicale 2020;21:257259.

14. Nouira N, Zouari A, Affes H, Gharbi Y, Hamdi D, Bahria W et al. Mise en place d'un circuit de patients suspects de COVID-19 et formation des soignants : le rôle primordial de la simulation. Pédagogie Médicale 2020;21:237-239.

15. Pierre-Duval P, Daveaux S, Hulot V, Fiani M. Adaptation d'un enseignement universitaire du Département de médecine générale d'Amiens lors de la crise sanitaire COVID-19. Pédagogie Médicale 2020;21:227-228.

16. Thibault L-P, Gariépy-Assal L, Moussa A. L'enseignement de la médecine au temps de la COVID-19 : le cas d'espèce de la pédiatrie. Pédagogie Médicale 2020;21:229-231.

17. Campillo-Paquet V, Demeester A, Laisney P. Crise COVID-19: évaluer à distance en contexte contraint. Rappels conceptuels et solutions alternatives. Pédagogie Médicale 2020;21:175-185.

18. Maherzi A, Ladner J, De Rouffignac S, Boelen C, Sylla C, Gresenguet $\mathrm{G}$ et al. Pandémie COVID-19: le défi de la responsabilité sociale des facultés de médecine. Pédagogie Médicale 2020;21:195-198.

19. Tenenbaum A, Bas A-C, Azogui-Levy S. Étudiants en odontologie, santé publique et crise sanitaire COVID-19. Pédagogie Médicale 2020;21:253-255. 
20. Sebbani M, Mansouri A, Soussi S, Alami Idrissi A, Thalal R, Safadi S et al. Pandémie COVID-19 et responsabilité sociale des étudiants en médecine en formation : quelles leçons pour l'avenir? (Cas de la Faculté de médecine de Marrakech). Pédagogie Médicale 2020;21:233-235.

21. Henriksen C, Jobin V, Deschênes M-F, Tremblay C, Charlin B, Fernandez N. Formation par concordance avec rétroaction multi-source aux questions qui émergent de la pratique médicale en contexte de pandémie COVID-19. Pédagogie Médicale 2020;21:203-205.

22. Organisation mondiale de la santé (OMS). Charte d'Ottawa pour la promotion de la santé. 1986. [On-line] Disponible sur: https://www.euro.who.int/__data/assets/pdf_file/ 0003/129675/Ottawa_Charter_F.p.pdf. 